\title{
Diseño de métricas basadas en estresores laborales para desarrolladores de software
}

\author{
Ma. Alejandra Zamudio ${ }^{1}$, Ramón R. Palacio ${ }^{2}$, Luis A. Castro ${ }^{3}$ \\ ${ }^{1}$ Instituto Tecnológico de Sonora, Unidad Nainari, \\ Sonora, México \\ ${ }^{2}$ Instituto Tecnológico de Sonora, Unidad Navojoa \\ Sonora, México
}

alejandra.aguilasocho@hotmail.com, luis.castro@acm.org ramon.palacio@itson.edu.mx

\begin{abstract}
Resumen. En este trabajo se investigaron los estresores laborales en desarrolladores de software con el objetivo de determinar un modelo de métricas que ayude a predecir y controlar los niveles de estrés. Para ello, se realizaron 10 entrevistas semiestructuradas a ingenieros en software y se analizaron los datos utilizando técnicas de teoría fundamentada. Los resultados muestran cuales son los principales estresores laborales en nuestros informantes. Además, se presenta un modelo constituido por cuatro métricas con sus respectivos ítems de medición: carga de trabajo, fatiga, demanda, y distracción laboral. Los resultados de este trabajo se pueden utilizar como base para la creación de herramientas creativas que permitan identificar patrones de comportamiento del desarrollador de software, de modo que ayuden a mejorar la productividad y disminuir las complicaciones psicológicas y biológicas que trae consigo el estrés durante el proceso de desarrollo de productos de software.
\end{abstract}

Palabras clave: estrés laboral, métricas de estrés, desarrolladores de software.

\section{Design of Metrics \\ Based on Work-related Stressors for Software Developers}

\begin{abstract}
This study investigated work-rated stressors in software developers aimed at determining a metric model to help predict and control stress levels. To this end, 10 semi-structured interviews were conducted with software engineers and data were analyzed using Grounded Theory techniques. The results of this work show the main work stressors in our informants. In addition, in this work, we present a model that consists of four metrics with their respective measurement items: workload, fatigue, demand, and work-distraction. The results of this work can be used as a basis for the creation of creative tools that allow the identification of behavioral patterns of the software developer, so they
\end{abstract}


can help improve productivity and reduce psychological and biological difficulties caused by stress during the software development process.

Keywords: work stress, stress metrics, software developers.

\section{Introducción}

El estrés laboral o también denominado ocupacional se refiere al nerviosismo, ansiedad y angustia relacionada con el trabajo, lo cual deriva en el sentimiento de un conjunto de reacciones emocionales, cognitivas, de comportamiento y biológicas que afectan a las personas [1]. Un estresor se refiere a "la condición o situación que provoca una respuesta negativa como la ira, frustración, ansiedad o tensión" [2]. Este concepto hace referencia al estímulo perturbador o amenazante que, tras su percepción y evaluación, genera una respuesta de estrés [3]. En este sentido, toda situación o condición presente en el medio externo, puede convertirse en un estresor laboral. En la industria del software, el estrés laboral adquirió mayor relevancia en los últimos años, a partir del incremento exponencial de la demanda ocasionada por los diferentes negocios que requieren adoptar continuas innovaciones tecnológicas para incrementar su ventaja competitiva [4, 5]. Esta exigencia ha generando notables cambios en el patrón de desgaste de los desarrolladores de software, provocando monotonía, aislamiento y colapso, las cuales son representaciones de estrés [6]. Como se menciona, el estrés laboral, es ocasionado por las exigencias que superan las habilidades, conocimientos y capacidades de los trabajadores para afrontar situaciones, el cuál repercute en la afectación de una gran cantidad de variables incluyendo productividad, eficiencia organizacional, salud física y mental [7]. En este sentido, el estrés laboral ha sido identificado como uno de los retos primordiales en el entorno empresarial actual, además de ser uno de los riesgos emergentes más importantes dentro de las organizaciones.

La influencia del estrés laboral en desarrolladores de software es una evidente realidad conocida a nivel mundial por diversas investigaciones que han demostrado los factores que atraen el estrés en los profesionales del software [2, 8, 9]. Además se ha determinado cómo es la relación entre los síntomas depresivos, el estrés ocupacional y las características del personal de TI $[4,10]$. Así como los efectos del estrés durante la generación de errores en el proceso de desarrollo de software [11]. A pesar de los múltiples estudios que existen en relación a este tema, ninguno de ellos se ha enfocado en establecer un conjunto de métricas que permitan identificar el grado de estrés entre los profesionales de software.

En México, el estrés ocupacional en desarrolladores de software ha sido poco examinado y de acuerdo a [12] el país representa el primer sitio de estrés ocupacional en el mundo, superando a China y Estados Unidos. Por otra parte, [13] reporta que todos los profesionales manifiestan algún síntoma de estrés laboral, además que el $60 \%$ sostiene altos niveles de éste. Por lo tanto, el interés de realizar este estudio surgió a partir de las evidentes dificultades que enfrentan los profesionales del software, las cuales repercuten en el desarrollo personal y profesional. Esto, debido a que día con 
día, el ambiente de trabajo resulta ser más dinámico y se trabaja bajo presión, razones por las cuales resulta crítico medir y disminuir el estrés, con el propósito de lograr una mejora en el rendimiento individual de los desarrolladores de software, condición que a su vez permitirá incrementar el rendimiento organizacional.

Por ende, el presente trabajo tiene como objetivo determinar un modelo de métricas que ayude a predecir los niveles de estrés en desarrolladores de software pertenecientes a organizaciones dedicadas a la producción de software. En consonancia con lo anterior, se pretende resolver el siguiente cuestionamiento: ¿Cuáles son las métricas que indican el grado de estrés de los trabajadores del software?

Para realizar esto es importante considerar a las métricas como un tipo de medida cuantificable, las cuales son utilizadas para tomar mejores decisiones [14]. Además sirven como instrumento fundamental para ayudar a los directores de proyectos a seleccionar los indicadores adecuados en torno al monitoreo del estrés de su equipo de trabajo. En referencia a lo anterior se afirma que son un medio esencial para entender, monitorear, controlar, predecir y probar el desarrollo de los equipos de trabajo de software [15].

El resto del documento se encuentra estructurado de la siguiente manera: En la sección II se presentan los trabajos relacionados al estrés laboral entre profesionales del software. La sección III detalla los pasos utilizados para construir y evaluar la solución. En las secciones IV y V se muestran los resultados y conclusiones del estudio.

\section{Trabajos relacionados}

Con la finalidad de identificar y entender la forma en que se ha abordado el estrés laboral en desarrolladores de software, en este apartado se presentan un conjunto de trabajos que describen algunos indicadores estresores que han sido percibidos en este tipo de trabajadores. Dado que resulta interesante tener una perspectiva general de esto y así categorizar dichos estresores acorde a su impacto en lo físico o psicológico del trabajador.

Diversos estudios se han enfocado en analizar el estrés ocupacional entre los profesionales de software. En [4] realizaron una investigación con el objetivo de examinar las relaciones entre el estrés laboral y los síntomas depresivos en los ingenieros y gerentes de software de una empresa japonesa. El instrumento de medición contempló ocho indicadores estresores subjetivos (sobrecarga de trabajo, falta de control, falta de recompensas, ambigüedad en el desarrollo profesional, conflictos con el equipo de trabajo, dificultad en la comunicación con los usuarios, cambio de TI y equipos de información inadecuados) y dos objetivos (horas de trabajo excesivas y promedio de horas frente al computador). Para los síntomas depresivos utilizaron escalas de depresión del centro de estudios epistemológicos (CES-D). La población de estudio fue de 1,694 ingenieros de software y 296 gerentes, todos de género masculino. Después de efectuar el análisis reportaron que los ingenieros tienen más falta de control de trabajo, menos sobrecarga laboral y cambios de tecnología informática que los gerentes. Además, los gerentes trabajan más horas pero usan menor cantidad de tiempo 
frente a una pantalla y tienen niveles de depresión más bajos en comparación con los ingenieros.

Por otro lado, [11] presentan una investigación cuantitativa con el objetivo de determinar los efectos del estrés sobre la generación de fallas durante el desarrollo de software. Como participantes eligieron a dos equipos de desarrollo conformados por dos programadores a quienes se les atribuyó realizar un sistema de gestión de bibliotecas bajo las mismas circunstancias excepto las metodologías de diseño. Uno de los equipos trabajó con diseño funcional mientras que el otro trabajó con diseño estructurado. Para esto utilizaron métricas psicológicas para evaluar el estrés mental ya que son fáciles de implementar. La métrica "presión por la fecha de entrega" fue la más eficaz, al revelar el nivel de estrés mental, mientras que la carga de trabajo tuvo la más alta correlación al número de fallas causadas. Para el estrés físico se usó una métrica llamada "condición física" que calculaba la afectación de dicho estrés. Después de aplicar métricas psicológicas y físicas para la medición de estrés durante el proceso de desarrollo se obtuvieron los siguientes resultados: los fallos de estrés representan una alta proporción de todas las fallas producidas, aunque el equipo que empleó la metodología diseño funcional tuvo mayor defectos que el de diseño estructurado; las fallas de naturaleza humana está correlacionada con los altos niveles de estrés; y por otro lado, el estrés físico lleva a cometer más fallas que el estrés mental.

En otro trabajo similar se examinó la relación entre los agentes estresores y las características del personal de TI de Singapur [10]. El instrumento fue elaborado mediante una extensa revisión bibliográfica y validado por expertos. La recolección de datos fue realizada a los trabajadores de TI pertenecientes a una empresa del ramo industrial mediante 257 encuestas electrónicas y 24 empleados del área de TI que participaron en cinco grupos focales con sesiones dirigidas de aproximadamente una hora y media. Tras hacer el análisis de los datos se dedujo que los principales factores que emergen como agentes estresores laborales son: la exigencia laboral, relaciones con el equipo de trabajo, preocupaciones, mantenimiento de sistemas, y ambigüedad de funciones y tareas administrativas.

Por otra parte, en [2] se desarrolló un instrumento para estudiar el estrés en los profesionales del software. Dicho cuestionario fue creado a partir del análisis de literatura existente e interacciones con académicos y empleados de software. Para este estudio se llevó a cabo el análisis factorial exploratorio a partir de 156 encuestas respuestas. Como resultados se identificaron que los principales factores que atraen estrés en los desarrolladores de software son: temor a la obsolescencia con el $13.1 \%$ de la varianza total, interacción con el equipo de trabajo $12.4 \%$, interacción con el cliente $7.2 \%$, interfaz trabajo-familia $6 \%$, sobrecarga de roles $5.5 \%$, cultura de trabajo $4.9 \%$, limitaciones técnicas $4.6 \%$, apoyo familiar hacia la profesión $3.7 \%$, exceso de trabajo $3.1 \%$ y riesgo al uso de nueva tecnología $3 \%$.

Otro estudio relacionado exploró los sucesos que generan estrés entre los profesionales de TI que asumen diversos roles en las compañías de Pakistán [8]. Dicha investigación se apoyó de técnicas cuantitativas para la recolección de datos a una población integrada por programadores, administradores de proyectos e ingenieros de calidad a través de un instrumento de medición que contenía los indicadores propuestos por [2]. Tras recabar los datos y efectuar el análisis se infiere que los factores no 
contribuyen de la misma forma entre los profesionales, a pesar de que las variables contempladas tienen el mismo peso, éstas impactan de manera diferente. Por lo que se encontró que el miedo a la obsolescencia, interacción con el cliente y carga de trabajo son los que más contribuyen al estrés del trabajador.

Además, [9] utilizó un enfoque cuantitativo con la finalidad de estudiar la relación entre las horas de exposición frente al computador y el estrés en los profesionales de TI. Veintiún ingenieros en software pertenecientes a empresas tecnológicas fue a quienes se les aplicó el cuestionario de ansiedad estado-rasgo (STAI) conformado por 20 indicadores de ansiedad/estado y 20 de ansiedad/rasgo. Al examinar los datos se encontró que estar expuesto más de ocho horas diarias frente a la computadora ocasiona mayor nivel de estrés a los trabajadores. Por lo tanto, existen relaciones positivas entre el nivel de estrés y el uso de la computadora.

Después de haber explorado los diferentes trabajos, es claro observar que aspectos relacionados a la cultura y exceso de trabajo son los que más fueron encontrados en la literatura, mientras que los menos considerados son el cambio de tecnología de información, síntomas depresivos, ansiedad y condición física. Dicho lo anterior, es importante mencionar que el nivel de consideración no es sinónimo del grado de incidencia, debido a que los indicadores impactan de manera diferente.

\section{Metodología}

Dada la naturaleza de la investigación se optó por utilizar una metodología cualitativa, puesto que el objetivo es entender la perspectiva de la población bajo estudio [16]. Para su elaboración se utilizaron como base técnicas de codificación abierta y codificación axial pertenecientes a la teoría fundamentada. Esta última se define como la teoría explicativa de un fenómeno en particular que emerge de los datos. $\mathrm{Su}$ finalidad es describir realidades, predecir sucesos, construir teorías o proposiciones no a priori [17].

\subsection{Participantes}

Se invitó a participar en entrevistas semiestructuradas a ingenieros en software pertenecientes a empresas localizadas en el municipio de Cajeme Sonora, México, dedicadas al desarrollo de software a la medida, implementación de software aeroespacial así como soporte y mantenimiento de sistemas. Los participantes fueron 10 ingenieros, entre ellos cuatro mujeres y seis hombres de edades entre 23 y 30 años. Nueve de estado civil solteros y uno casado. Con experiencia laboral menor a tres años.

Resulta oportuno destacar que el sector de software en México cuenta con importantes fortalezas que lo ubican como un claro centro de atracción de inversiones en el mercado global. Actualmente ocupa el octavo lugar mundial como mejor destino para proveer servicios de tecnologías de información [18]. En el periodo 2010-2015 esta industria representó una tasa de crecimiento promedio anual de 13\% [19]. Además, desde el año 2008 a la fecha han generado más de 7,000 mil empleos que representan un elevado impacto en el desarrollo tecnológico y social del país [20]. 


\subsection{Materiales $\mathbf{y}$ métodos}

La técnica empleada para recopilar datos fue entrevistas semiestructuradas ya que permiten al entrevistador estar preparado y otorga a los informantes amplia libertad de expresión [21]. Para esto se utilizó un protocolo de entrevista con preguntas abiertas previamente elaboradas que abordaban los siguientes temas:

Incompetencia laboral: Los cuestionamientos de este tema se basan en analizar aspectos relacionados con el estrés que provoca el aprendizaje y la adaptación respecto a las nuevas tecnologías y mejores prácticas de desarrollo.

Interacción con el equipo de trabajo: Incluye interrogantes para conocer el trato que recibe el desarrollador de software, la existencia de rivalidades, discriminación o favoritismo.

Interacción con el cliente: Las interrogantes agrupadas en este apartado tienen la finalidad de investigar cómo es la relación que encara el desarrollador durante la interacción con los clientes en el proceso de desarrollo de software.

Carga laboral: Se encarga de indagar la cantidad de actividades asignadas a los desarrolladores de software, y la percepción del estrés que puede causarle.

Ambiente laboral: Se cuestiona la percepción del desarrollador de software sobre su ambiente de trabajo y los aspectos relacionados que son causantes de estrés.

Las entrevistas fueron grabadas con consentimiento previo, sin interrupciones, las cuales tuvieron duraciones entre 50 y 53 minutos. Después, se procedió a su transcripción textual para su análisis.

\subsection{Procedimiento de análisis}

Para el análisis se utilizó teoría fundamentada con la finalidad de detectar patrones en los datos. El análisis utilizando teoría fundamentada involucra tres principales etapas:

1. Codificación abierta, donde se analizó cada renglón de las transcripciones buscando conceptos, acciones o enunciados que ayuden a describir los estresores laborales. A la información encontrada se le asignó un código de clasificación, los cuales fueron agregados a una lista que fue depurada al final de hacer la codificación, dejando aquellos que tienen una fuerte relación con el estrés en los desarrolladores de software.

2. Codificación axial, la cual consistió en examinar cada uno de los códigos, buscando similitudes para agruparlos conforme a sus circunstancias y condiciones. A cada agrupación se le asignó una propiedad que los definiera para determinar la categoría. Posteriormente se procedió en determinar los factores críticos con la finalidad de conocer los elementos que intervienen en el estrés de los profesionales del software, a partir de ello se elaboraron el conjunto de métricas. 
3. Codificación selectiva, consistió en integrar y elegir las categorías para delimitar la teoría.

\section{Resultados y discusiones}

En este apartado se presentan los resultados del análisis de las entrevistas, centrados principalmente en la pregunta de investigación: ¿Cuáles son las métricas que indican el grado de estrés de los trabajadores del software?, planteada en la sección I.

\section{A. Estresores laborales}

Este análisis inició con la identificación de los principales estresores laborales, los cuales responden parcialmente a la pregunta de investigación. Por lo que a continuación se detallan cada uno de ellos:

Sobrecarga laboral. Este estresor hace hincapié en sobrepasar el límite de requerimientos que un desarrollador de software es capaz de resolver en un determinado tiempo. Por ejemplo, el Participante 6 comenta: “...me estreso en particular cuando me asignan mucha carga de tareas y muy poco tiempo para realizarlas" (Participante 6). De manera similar, el Participante 3 explica: “...es frustrante tener muchos requerimientos por resolver y sentirme incapaz de terminarlos a tiempo" (Participante 3). En ese sentido, uno de los aspectos que parecen influir en niveles de estrés puede ser el número de tareas, porque pueden ser demasiadas a realizar en un corto plazo. Sin embargo, es interesante cómo el Participante 3 hace referencia a su capacidad para realizarlas, ya que el sentirse abrumado por el número de tareas puede tener un efecto negativo en su percepción de capacidad para realizar su trabajo.

Organización en la fase final del proyecto. Se refiere a la administración inconsistente de las actividades respecto a la fecha de entrega parcial o total del proyecto al cliente. En relación a este estresor el Participante 10 expresa: "...siempre que estoy en la fase final del proyecto siento que mi rendimiento es bajo, porque tengo el tiempo encima y muchos requerimientos pendientes por resolver" (Participante 10). Además el Participante 4 argumenta: “...la mala organización del tiempo en las actividades provoca en mi niveles altos de frustración durante la fase final del proyecto" (Participante 4). Respecto a lo antes mencionado, es claro observar que al tener inadecuada organización durante el proyecto se podrían traer consecuencias en los resultados u "outputs" del desarrollador durante la fase final. No obstante, puede causar afectaciones psicológicas en el individuo, tal es el caso del Participante 4, quien percibe sentimientos de ira y decepción.

Complejidad de actividades. Representa la dificultad que constituye a un algoritmo de programación. Surge a partir de los inconvenientes que tiene que superar el desarrollador de software para concluir la actividad. Como muestra de ello, el participante 2 manifiesta: “...en el proyecto pasado cuando no me tocaba hacer 
las tareas difíciles me causaba alegría porque sabía que no batallaría para terminarlas, pero cuando me tocaba realizarlas era preocupante y me estresaba mucho porque no sabía sí iba a terminarlas en tiempo y forma" (Participante 2). Esto, nos revela que un parámetro interesante a considerar en la asignación de tareas complejas es el tiempo, ya que desde antes de iniciar la tarea puede agobiar al desarrollador.

Incompetencia laboral. Se refiere a la necesidad de poseer las competencias necesarias para el desempeño exitoso. Los desarrolladores de software relacionan este estresor con la adaptación, aprendizaje e implementación de nuevas tecnologías y buenas prácticas de desarrollo. Para ejemplificar esto, el Participante 9 argumenta: “...es indispensable para mi estar en constante actualización respecto a las nuevas tecnologías y mejores prácticas de desarrollo para poder estar a la vanguardia profesional y brindarles todo lo que la compañía y los clientes requieren" (Participante 9). Con este comentario, el participante percibe la necesidad de aumentar sus conocimientos y habilidades para tener aumentar capacidad productiva.

Actividades no preferidas. Este estresor representa las actividades que un desarrollador de software no disfruta realizar. Por ejemplo, el Participante 1 dice: “...realizar la documentación u otras actividades aburridas, simplemente me ocasiona estrés, distracción, cansancio y frustración porque no disfruto lo que estoy haciendo" (Participante 1). En este sentido, el participante percibe estas actividades como causantes de respuestas psicológicas negativas, al mismo tiempo pueden ocasionarle distracciones.

\section{B. Métricas}

A partir de la identificación de estresores, se diseñó un modelo de métricas que tienen como objetivo medir el estrés en desarrolladores pertenecientes a la industria del software. Acorde a lo anterior, la Tabla 1 muestra cuatro métricas propuestas con sus respectivos ítems de medición, los cuales se describen a continuación

Carga de trabajo: consta de seis ítems relacionados con la cantidad, duración y tamaño de las tareas asignadas a un desarrollador. Tiene la finalidad de estimar el número de requerimientos óptimos que un desarrollador puede realizar sin sobrepasar su carga laboral.

Fatiga laboral: su propósito es medir la complejidad de actividades e incompetencia laboral, por lo tanto, ésta métrica se integra de seis ítems que abordan información relacionada al sobreesfuerzo o tensión sostenida por un desarrollador de software.

Demanda laboral: tiene el objetivo de contrarrestar el estrés respecto a la falta de organización en la fase final del proyecto, para ello se incluyen dos ítems relacionados con el tiempo requerido, disponible y adicional demandando por el desarrollador de software. 
Distracción laboral: métrica conformada por tres ítems vinculados al estresor actividades no preferidas. Su fin es determinar tareas que ocasionan estrés al desarrollador relacionadas con la apatía.

Tabla 1. Métricas para medir el estrés en desarrolladores de software.

\begin{tabular}{|c|c|c|c|}
\hline Métrica & Ítems & Definición del ítem & $\begin{array}{l}\text { Estresores } \\
\text { afectados }\end{array}$ \\
\hline \multirow{6}{*}{$\begin{array}{l}\text { Carga de } \\
\text { trabajo }\end{array}$} & Tareas asignadas & Número de tareas impuestas & \multirow{6}{*}{$\begin{array}{l}\text { Sobrecarga } \\
\text { laboral }\end{array}$} \\
\hline & $\begin{array}{l}\text { Tasa de llegada de la } \\
\text { tarea }\end{array}$ & $\begin{array}{l}\text { Número de tareas impuestas en una unidad } \\
\text { de tiempo }\end{array}$ & \\
\hline & Tamaño de la tarea & $\begin{array}{l}\text { Cantidad de actividades necesarias para } \\
\text { completar la tarea }\end{array}$ & \\
\hline & $\begin{array}{l}\text { Tiempo destinado a } \\
\text { completar la tarea }\end{array}$ & Duración para completar la tarea & \\
\hline & $\begin{array}{l}\text { Tiempo invertido en } \\
\text { completar la tarea }\end{array}$ & Duración empleada para completar la tarea & \\
\hline & Prioridad de la tarea & $\begin{array}{l}\text { Nivel de importancia } \\
\text { Nivel de urgencia }\end{array}$ & \\
\hline \multirow{6}{*}{$\begin{array}{l}\text { Fatiga } \\
\text { laboral }\end{array}$} & $\begin{array}{l}\text { Complejidad ciclo- } \\
\text { mática de la tarea }\end{array}$ & $\begin{array}{l}\text { Número de caminos independientes dentro } \\
\text { de un fragmento de código }\end{array}$ & \multirow{6}{*}{$\begin{array}{c}\text { Complejidad } \\
\text { de actividades } \\
\text { Incompetencia } \\
\text { laboral }\end{array}$} \\
\hline & $\begin{array}{l}\text { Conocimiento } \\
\text { técnico }\end{array}$ & $\begin{array}{l}\text { Porcentaje de conocimientos técnicos } \\
\text { relacionados al proyecto }\end{array}$ & \\
\hline & $\begin{array}{l}\text { Autoaprendizaje } \\
\text { técnico }\end{array}$ & $\begin{array}{l}\text { Porcentaje de aprendizaje adicional } \\
\text { involucrado durante la ejecución de la tarea }\end{array}$ & \\
\hline & $\begin{array}{l}\text { Información de la } \\
\text { tarea }\end{array}$ & $\begin{array}{l}\text { Porcentaje de claridad en cuanto a metas y } \\
\text { objetivos de la tarea }\end{array}$ & \\
\hline & $\begin{array}{l}\text { Incertidumbre de la } \\
\text { tarea }\end{array}$ & $\begin{array}{l}\text { Porcentaje de información necesaria } \\
\text { proporcionada para la actividad }\end{array}$ & \\
\hline & Número de bugs & Número de defectos en el software & \\
\hline \multirow{2}{*}{$\begin{array}{l}\text { Demanda } \\
\text { laboral }\end{array}$} & $\begin{array}{l}\text { Demanda temporal } \\
\text { del proyecto }\end{array}$ & $\begin{array}{l}\text { Tiempo requerido para terminar la tarea y el } \\
\text { disponible }\end{array}$ & \multirow{2}{*}{$\begin{array}{l}\text { Organización } \\
\text { en la fase final } \\
\text { del proyecto }\end{array}$} \\
\hline & $\begin{array}{l}\text { Horas extra de } \\
\text { trabajo }\end{array}$ & Porcentaje de horas extra semanales & \\
\hline \multirow{3}{*}{$\begin{array}{l}\text { Distracción } \\
\text { laboral }\end{array}$} & $\begin{array}{l}\text { Interrupciones de la } \\
\text { tarea }\end{array}$ & $\begin{array}{l}\text { Número de veces en que se suspende la } \\
\text { ejecución de la tarea }\end{array}$ & \multirow{3}{*}{$\begin{array}{l}\text { Actividades no } \\
\text { preferidas }\end{array}$} \\
\hline & $\begin{array}{l}\text { Duración de la } \\
\text { interrupción }\end{array}$ & $\begin{array}{l}\text { Tiempo que transcurre desde el inicio al fin } \\
\text { durante la interrupción }\end{array}$ & \\
\hline & $\begin{array}{l}\text { Frecuencia de } \\
\text { interrupciones }\end{array}$ & $\begin{array}{l}\text { Número de veces que sucede la interrupción } \\
\text { de la tarea }\end{array}$ & \\
\hline
\end{tabular}

La mayoría de los factores estresores identificados ya han sido discutidos en estudios similares con diversas semejanzas. En su trabajo [2] presentaron un listado de factores estresores similares a los identificados en esta investigación. Tal vez la diferencia más marcada tiene que ver con la aparición de un nuevo factor que ellos no contemplaron, el cual está relacionado con las actividades no preferidas por los desarrolladores. Por otro lado [4, 8-11], ha dejado demostrado que la sobrecarga laboral es una de las circunstancias más generadoras de estrés. Respecto al estresor organización en la fase final del proyecto en [2] determinaron que la presión por la fecha de entrega es eficaz al momento de revelar niveles de estrés mental. A pesar que diversos autores han 
orientado sus esfuerzos en determinar cuáles son los factores estresores más influyentes en desarrolladores de software, ninguno de ellos se enfocó en diseñar métricas que ayuden a identificar el grado de estrés entre los profesionales.

\section{Conclusiones}

En este trabajo se presentaron los resultados de un estudio cualitativo sobre el diseño de métricas que permiten controlar los factores estresores en los desarrolladores de software. Los resultados serán utilizados como base para la creación de herramientas creativas que permitan determinar patrones de comportamiento, de modo que ayuden a que los desarrolladores a ser más productivos y disminuir las complicaciones psicológicas y biológicas que trae consigo el estrés durante el proceso de desarrollo de productos de software, a su vez con dichos indicadores se podrá mostrar el nivel de estrés de cada uno de ellos.

Por otro lado, estas métricas pueden ser utilizadas por los directores de proyectos de software para crear mecanismos apropiados relacionados con la selección de tareas adecuadas para tener un mejor rendimiento por parte de su equipo de trabajo. Como trabajo futuro, se pretende evaluar con un caso de estudio práctico el estrés de los desarrolladores de software utilizando las métricas planteadas anteriormente, con la finalidad de determinar patrones de comportamientos y crear estrategias para ayudarlos a que trabajen de una forma más confortable. El estudio se llevó a cabo sólo con ingenieros en software, razón por la cual los resultados deben interpretarse con precaución, además fue un estudio de tipo exploratorio transversal y está limitado en su capacidad de interpretaciones causales.

\section{Referencias}

1. Singh, J., Pratap, B., Narban, S., Singh, J.: A Conceptual Study on Occupational Stress (Job Stress/Work Stress) and its Impacts. Jaipur (Rajasthan) 2 MSc (CS), pp. 2395-4396 (2016)

2. Rajeswari, K. S., Anantharaman, R. N.: Development of an Instrument to Measure Stress Among Software Professionals: Factor Analytic Study. In: Proceedings of the 2003 SIGMIS Conference on Computer Personnel Research: Freedom in Philadelphialeveraging Differences and Diversity in the IT Workforce, ACM, New York, NY, USA, pp. 34-43 (2003)

3. Durán, M.M.: Bienestar psicológico: el estrés y la calidad de vida en el contexto laboral. Revista nacional de administración 1, pp. 71-84 (2010)

4. Haratani, T., Fujigaki, Y., Asakura, T.: Job stressors and depressive symptoms in Japanese computer software engineers and managers. Advances in Human Factors (1995)

5. Laudon, K. C., Laudon, J. P.: Management information systems : Managing the Digital Firm. 9th edition, pp. 103-105 (2007)

6. Velázquez, A.: Mujer, trabajo y estrés. Revista latinoamericana de salud en el trabajo (2001) 
7. Chilton, M. A., Hardgrave, B. C., Armstrong, D. J.: Person-Job Cognitive Style Fit for Software Developers: The Effect on Strain and Performance. Journal of Management Information Systems 22, pp. 193-226 (2005)

8. Rashidi, Z., Jalbani, A. A.: Job Stress among Software Professionals in Pakistan: A Factor analytic Study. J Independent Stud Res (MSSE), 7, pp. 1-12 (2009)

9. Sushma, S. S.: Influence of Hours of Computer Usage on Stress in Software Professionals. Journal of Evolution of Medical and Dental Sciences, 3, pp. 9621-9626 (2014)

10. Lim, V., Teo, T.: Occupational stress and IT personnel in Singapore: factorial dimensions and differential effects. International Journal of Information Management (1999)

11. Furuyama, T., Arai, Y., Iio, K.: Analysis of fault generation caused by stress during software development. Journal of Systems and Software (1997)

12. Villaseñor, N.: Te sientes quemado o desgastado por el trabajo? Es el síndrome de burnout. In: México Ciencia y Tecnología. Recuperado de: http://conacytprensa.mx/index.php/ ciencia/salud/6398-sindrome-de-burnout-reportajefinal (2016)

13. Prado J Estudio confirmatorio de la escala mexicana de desgaste ocupacional (EMEDO): Un instrumento de burnout para mexicanos. Revista Interamericana de Psicología. (2016)

14. Pfleeger, S. L.: Assessing measurement. IEEE Software, Vol. 14, No. 25 (1997)

15. Serrano, M., Piattini, M., Calero, C.: Un método para la definición de métricas de software. In: 1er Workshop en Métodos de Investigación y Fundamentos filosóficos en Ingeniería del Software y Sistemas de Información, pp 65-74 (2002)

16. Olabuénaga, J.: Metodología de la investigación cualitativa (2012)

17. Strauss, A., Corbin, J.: Bases de la investigación cualitativa: técnicas y procedimientos para desarrollar la teoría fundamentadal (2002)

18. Kearney, A. T.: A.T. Kearney Releases 2016 Global Services Location Index (2016)

19. PROMÉXICO: Servicios de TI y software en México (2016)

20. PROMÉXICO: ProMéxico e industria de tecnologías de la información y comunicaciones desarrollan plan de trabajo para fortalecer al país (2017)

21. Longhurst, R.: Semi-structured interviews and focus groups. Key methods in geography (2003) 